\title{
Classification of EEG Physiological Signal for the Detection of Epileptic Seizure by Using DWT Feature Extraction and Neural Network
}

\author{
Manisha Chandani, Arun Kumar \\ Department of Electronics \& Telecommunication, Chhattisgarh Swami Vivekananda Technical University, Durg, India
}

Email address:

chandani.manisha09@gmail.com (M. Chandani), arun.kumar@bitdurg.ac.in (A. Kumar)

\section{To cite this article:}

Manisha Chandani, Arun Kumar. Classification of EEG Physiological Signal for the Detection of Epileptic Seizure by Using DWT Feature Extraction and Neural Network. International Journal of Neurologic Physical Therapy. Vol. 3, No. 5, 2017, pp. 38-43. doi: 10.11648/j.ijnpt.20170305.11

Received: June 28, 2017; Accepted: July 17, 2017; Published: October 24, 2017

\begin{abstract}
EEG (Electroencephalogram) is a technique for identifying neurological disorders. There are various neurological disorders like Epilepsy, brain cancer, etc. Feature extraction and classification of electroencephalogram (EEGs) signals for (normal and epileptic) is a challenge for engineers and scientists. Various signal processing techniques have already been proposed for classification of non-linear and non stationary signals like EEG. In this work, neural network analysis (NNA) based classifier was employed to detect epileptic seizure activity from background electro encephalographs (EEGs). Two types of EEG signals (healthy subject with eye open condition, epileptic) were selected for the analysis. Signals were reprocessed, decomposed by using discrete wavelet transform DWT till 5th level of decomposition tree. Various features like mean. Standard deviation, median, entropy, kurtosis and skewness were computed and consequently used for classification of signals. The range of these features in non-epileptic and epileptic group of 80 subjects each from data set is analyzed for data available at the Department of Epileptology, University of Bonn, and the parameters with distinct non-overlapping zone are identified. The results show the promising classification accuracy of nearly $100 \%$ in detection of abnormal from normal EEG signals. The main purpose of this new approach is that the computation time of NNA classifier is less to provide better accuracy. This proposed classifier can be used to design expert system for epilepsy diagnosis purpose in various hospitals.
\end{abstract}

Keywords: Electroencephalogram (EEGs), Discrete Wavelet Transform (DWT), Principal Component Analysis (PCA), Neural Network Analysis (NNA), Epileptic, Seizure

\section{Introduction}

Electroencephalogram is the study of the electrical signals produced by brain. Electrical signals generated by the human brain represent the thinking of the mind and the status of the body. The close study of those EEG (EEG) signals is helpful in several analysis areas like detection and classification of event connected potentials, seizure detection and prediction, brain-computer interfacing, Study of mental disorders like psychiatrically disorders, dementedness and sleep signal analysis. For higher understanding of human behaviour the EEG signal waves square measure more divided in five major sub-bands supported the frequency ranges. These bands from low to high frequencies respectively are called delta $(\delta)$ (Range $0.5-4 \mathrm{~Hz}$ ), theta $(\theta)($ Range $4-8 \mathrm{~Hz}$ ), alpha $(\alpha)$
(Range 8-13 Hz), beta $(\beta)$ (Range 13-30 Hz), and gamma $(\gamma)$ (Range 30-45 HZ) [1]. The visual distinction of seizure from common artefacts among associate degree graph measure is predicated on the form and spikiness of the waveforms. A proof with seizure has a tripping and distinguished peaky, whereas the foremost of alternative artefacts square measure non-stationary and every which way formed. However considering the actual fact that the recorded EEG pattern may be a special mapping of signals captured by placement of electrodes onto totally different regions of the scalp, it's very troublesome for person to watch and perceive the particular behaviour of the brain by just visual review. Therefore there's associate degree ever increasing demand of simply accessible and absolutely automatic convulsion detection system mistreatment EEG signals. During this paper we have a 
tendency to propose options extraction of EEG signals from Discrete wavelet transforms (DWT). Further, Neural Network Analysis (NNA) is employed for classification into 2 categories i.e. is epileptic and normal. So as to scale back the time and space complexity and to avoid redundancy within the ascertained options, we've applied Principal element Analysis (PCA).

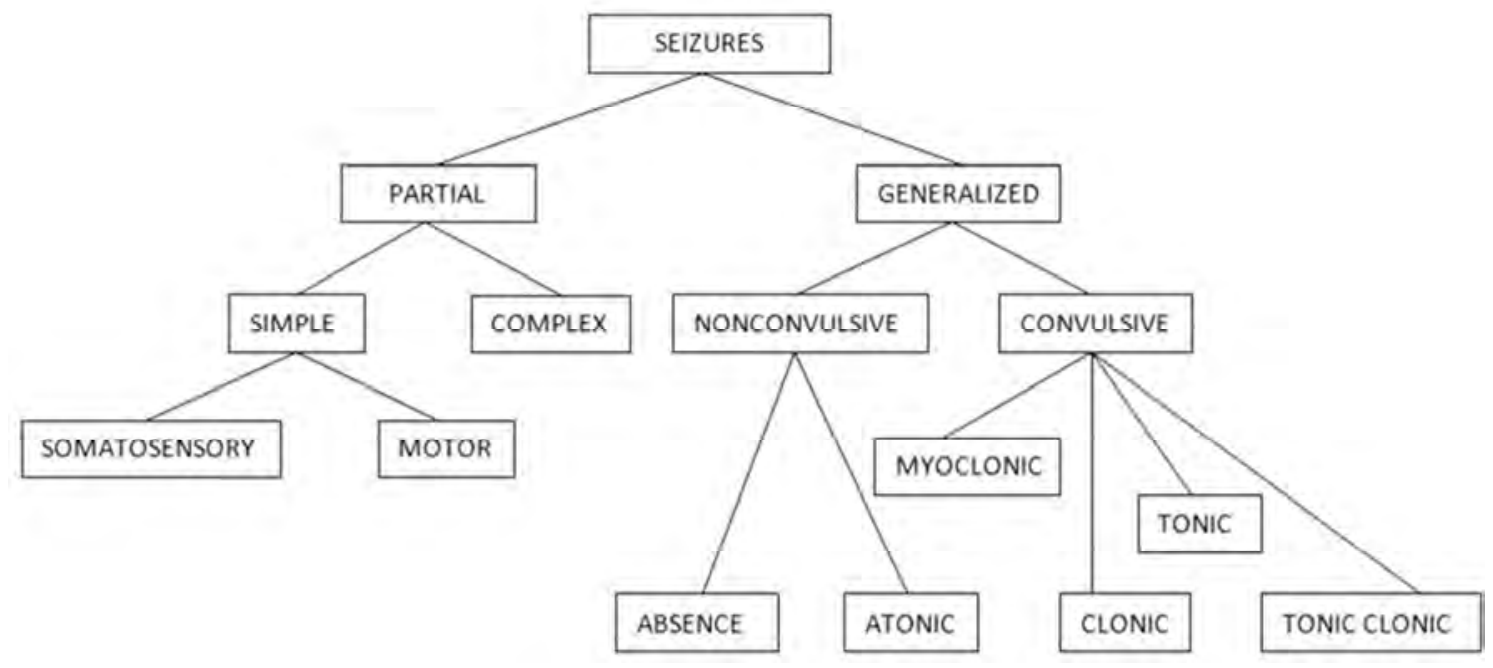

Figure 1. Classification of Epileptic Seizure.

\section{Related Work}

The electrical signals for brain activity were first recorded by the English scientist Richard Caton in 1875. Hans Berger started the study of EEGs from human brain in 1920 [2]. Epilepsy is a Greek word, which means 'to seize or attack'. The very basic concepts of epilepsy can be found in ancient Indian medicine (4500-1500 BC) as apasmara, which means "loss of consciousness". Babylonian tablet in the British Museum in London also gives the detailed knowledge about the epileptic disease and its cure [1]. Kaufman associated the epileptic attacks with abnormal electrical discharges [3]. Most of the epilepsy analysis methods developed in the 20th century were based on the concept of visual inspection of EEG signals by highly skilled electroencephalography. However, with the advancement in the field of signal processing and pattern recognition, different automatic techniques of epileptic seizure detection have been developed in last two decades [6], [9]. Spectral analysis based feature extraction method provides poor results for EEG classification as the frequency domain information is provided at the cost of time domain information such as the amplitude distribution and EEG pattern. Hence, both time and frequency domain based feature extraction algorithms such as Discrete Wavelet Transform (DWT) are being used in current research [4]-[6]. The other advantage of DWT over spectral analysis is its suitability for analysis of nonstationary signals like EEG [7], [8]. Kai Fu et al. Have recently published their work with Hilbert-Huang Transformed (HHT) based approach [9].

\section{Data Selection and Recording}

We have used publicly available data described in [10].
The complete data set consists of five sets (denoted A-E) each containing 100 single-channel EEG segments. These segments were selected and cut out from continuous multichannel EEG recordings after visual inspection for artefacts, e.g., due to muscle activity or eye movements. Sets A and B consisted of segments taken from surface EEG recordings that were carried out on five healthy volunteers using a standardised electrode placement scheme.
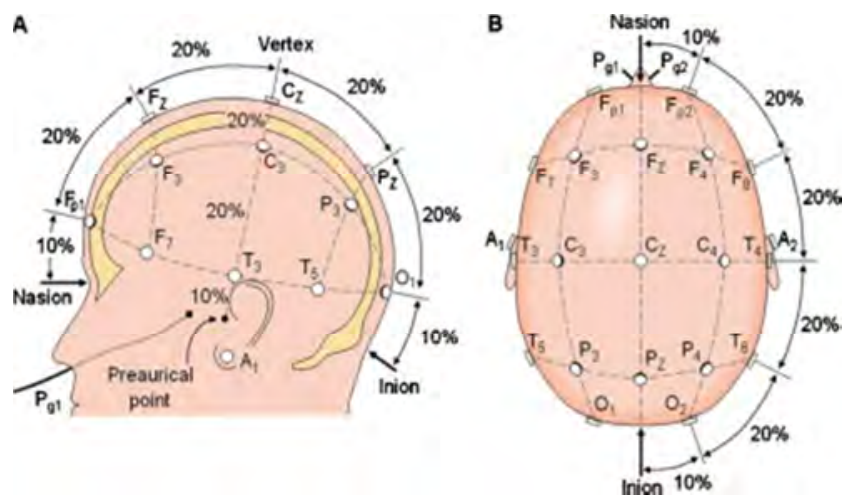

Figure 2. The 10-20 international system of electrode placement c images of normal and abnormal cases.

Volunteers were relaxed in associate awake-state with eyes open (A) and eyes closed (B), severally. Sets C, D, and E originated from graph archive of pre-surgical identification. EEGs from 5 patients were hand-picked, all of whom had achieved complete seizure management when surgical operation of one of the hippocampal formations, that was thus properly diagnosed to be the epileptogenic zone. Segments in set D were recorded from among the epileptogenic zone, and people in set $\mathrm{C}$ from the hippocampal formation of the other hemisphere of the brain. whereas sets $\mathrm{C}$ and D contained solely activity measured 
throughout seizure free intervals, set E solely contained seizure activity. The electrodes were set as shown in fig. 1 . The frontal lobe, temporal lobe, parietal lobe, central lobe, and occipital lobe were named F, T, P, C, and O, respectively [11].

Here segments were selected from all recording sites exhibiting ictal activity. All EEG signals were recorded with the same 128-channel amplifier system, using an average common reference. The data were digitised at 173.61 samples per second using 12 bit resolution. Band pass filter settings were $0.53-40 \mathrm{~Hz}(12 \mathrm{~dB} / \mathrm{oct})$. In this study, we used two dataset (A and $\mathrm{E}$ ) of the complete dataset.
Decomposition of Non-epileptic and Epileptic EEG signal with delta, theta, alpha, beta and gamma subband decomposition is shown in Figure 3 and 4 with decomposition frequency of each subband. According to the Nyquist Theorem, the maximum useful sampling frequency is half of the sampling frequency. Here we have:

$$
\frac{f_{S}}{2}=\frac{173.61}{2}=86.6 \mathrm{~Hz}
$$

Where $f_{s}$ is the sampling frequency. Figure no. 3 shows the five level decomposition of EEG data sample.

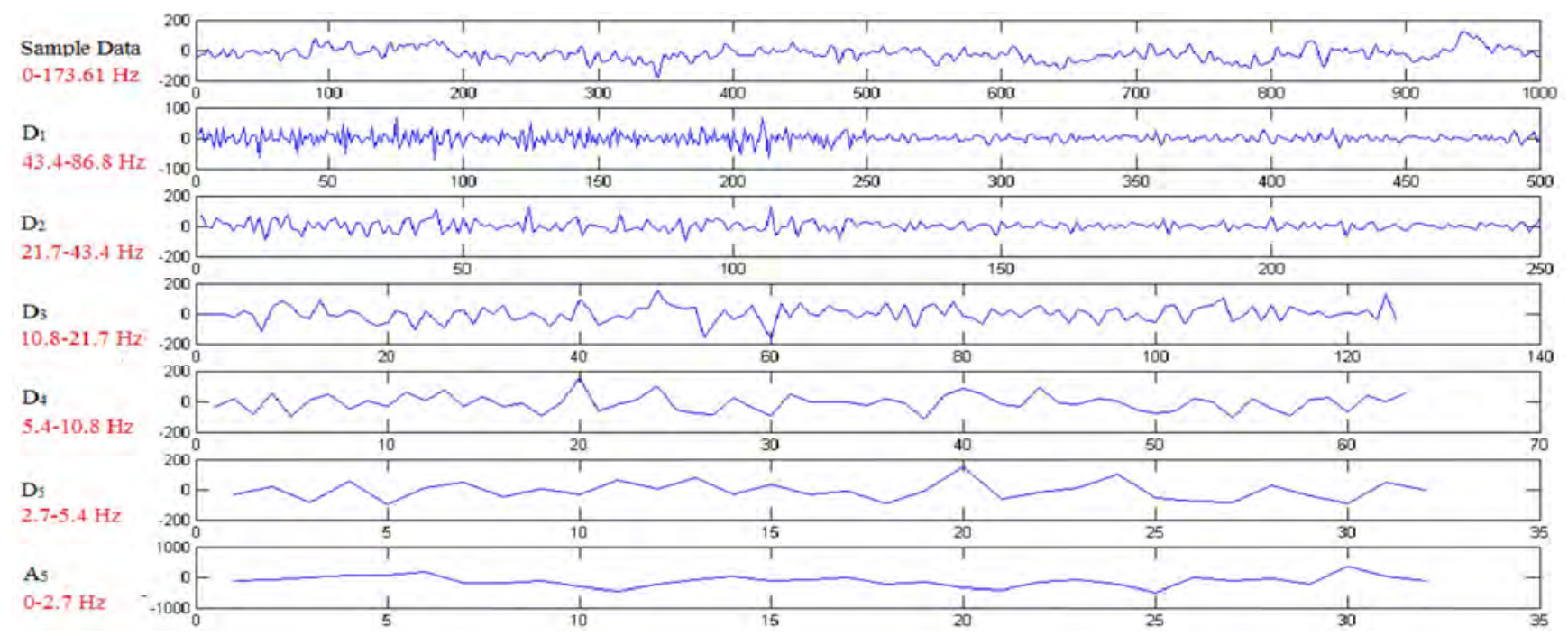

Figure 3. Level wavelet decomposition of sample data (from set A) signals of 0-173.61 Hz. D1-D5 are details and A5 is approximation. (For clear visibility only 1000 initial samples taken from 4097 samples of the sample data and axis are not equalon the sub-plots).

\section{Methodology}

\subsection{Wavelet Transform for Signal Analysis}

We used Wavelet transform technique to decompose the signals and reconstruct the data accurately. rippling remodel technique could be a appropriate analytical tool for the Non Stationary signals analysis like encephalogram and it had been projected by Jean Morlet a French geologist in 1982[12]. It involves the breaking down of the brain signals into numerous shorter reads of bands as per demand. In distinct wavelet analysis, a multi-resolution description is employed to decompose a given signal $\mathrm{f}(\mathrm{t})$ into progressively finer details supported 2 sets of basic functions [13] the wavelets and the scaling functions, as follows:

$$
f(t)=\sum_{\mathrm{i}=\mathrm{z}} 2^{\frac{j}{2}} C_{j}(k) \varphi\left(2^{j} t-k\right) \sum_{j=0}^{j-1} \sum_{k=0}^{\infty} 2^{j} d_{j} \psi\left(2^{j} t-k\right)
$$

where functions. $\varphi(\mathrm{t})$ and $\psi(\mathrm{t})$ are the basic scaling and mother wavelet respectively. Five sub-bands are obtained, namely Delta $(0-4.05 \mathrm{~Hz})$, Theta $(4.05-8.1 \mathrm{~Hz})$, Alpha $(8.1-$ $12.15 \mathrm{~Hz})$, Beta $(12.15-32.5 \mathrm{~Hz})$ and Gamma $(>32.5 \mathrm{~Hz})$ [14].

The number of decomposition levels is chosen supported the dominant frequency parts of the signal. The amount square measure chosen such those components of the signal that correlates well with the frequencies necessary for classification of the signal square measure maintained within the riffle coefficients. Within the present study, since the encephalogram signals don't have any helpful frequency parts higher than 30 cycles/second, the amount of decomposition levels was chosen to be four.

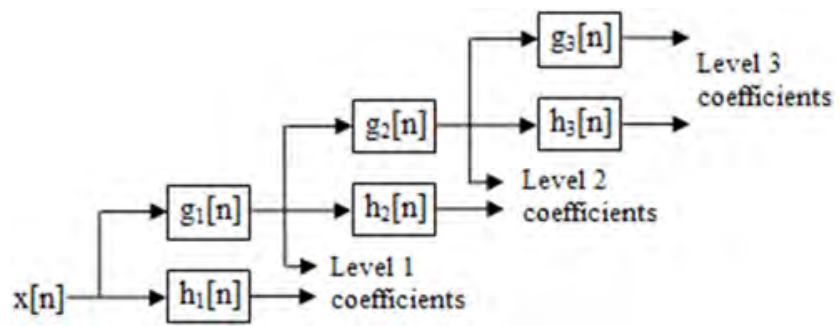

Figure 4. Sub-band decomposition of DWT implementation; $h[n]$ is the high pass filter, $g[n]$ the low-pass filter.

Thus, the encephalogram signals were rotten into details D1-D4 and one final approximation, A4. Usually, tests square measure performed with differing kinds of wavelets and also the one, which supplies most potency, is chosen for the actual application. The smoothing feature of the Daubechies riffle of order a pair of (db2) created it additional applicable to sight changes of encephalogram signals. Hence, 
the riffle coefficients were computed victimization the $\mathrm{db} 4$ within the present study. The projected methodology was applied on each knowledge set of encephalogram knowledge (Sets A and E).

\subsection{Feature Extraction}

In this work we have chosen the statistical parameters All the extracted features constitute the combined feature index $(\mathrm{CFI})=(\mathrm{F} 1, \mathrm{~F} 2, \mathrm{~F} 3, \mathrm{~F} 4, \mathrm{~F} 5 \ldots . . . \mathrm{Fn})$, which is presented as an input to the ANN network. The features used in evaluating the performance of the proposed scheme are mean, standard deviation, median; entropy, kurtosis and skewness were calculated at each decomposition level starting from 05 to 08 for the normal and epilepsy categories of signals. The mathematical equations are represented as follows:

\section{Mean $\mu$}

Mean are fundamental statistical attributes of a time series the arithmetic mean of a time series is the average expected value of that time series. In some cases, the mean value of a time series can be the operating point or working point of a physical system that generates the time series.

$$
\begin{aligned}
\mu & =\frac{1}{\mathrm{~N}} \sum_{\mathrm{i}=1}^{\mathrm{N}} \mathrm{A}_{\mathrm{i}} \\
i & =1,2,3,4 \ldots \ldots \ldots . .
\end{aligned}
$$

The mean indicated by $\mu$. The Value in the signal $\mathrm{X}$, by letting the index, $\mathrm{i}$, run from 0 to 1 . Then finish the calculation by dividing the sum by $\mathrm{N}$. This is identical to the equation: $\mu=\left(X_{0}+X_{1}+X_{2}+\ldots+X_{N-1}\right) / \mathrm{N}$.

\section{Standard Deviation $\boldsymbol{\sigma}$}

The standard deviation is similar to the average deviation except the averaging is done with power instead of amplitude. This is achieved by suaring each of the deviation before taking the average. To finish the square root is taken to compensate for the intial squaring. In equation from the standard deviation is calculation.

$$
\begin{gathered}
\sigma=\sqrt{\frac{1}{\mathrm{~N}} \sum_{\mathrm{i}=1}^{\mathrm{N}}\left(\mathrm{x}_{\mathrm{i}}-\psi_{\mathrm{i}}\right)^{2}} \\
i=1,2,3,4 \ldots \ldots \ldots
\end{gathered}
$$

$\mathrm{X}$ is signal with mean $\mu, \mathrm{N}$ is the number of sample and $\sigma$ is standard deviation.

\section{Kurtosis $k$}

The kurtosis are higher-order statistical attributes of a time series. Kurtosis measures the peakedness of the probability density function (PDF) of a time series. A kurtosis value close to three indicates a Gaussian-like peakedness. PDFs with relatively sharp peaks have kurtosis greater than three. PDFs with relatively flat peaks have kurtosis less than three.

$$
\mathrm{k}=\frac{\mathrm{E}(\mathrm{x}-\mu)^{4}}{\sigma^{4}}
$$

Where $\mathrm{X}$ is a signal, $\mu$ is the mean of $x, \sigma$ is the standard deviation of $\mathrm{X}$, and $E(t)$ represented the expected value of the quantity $\mathrm{t}$ Kurtosis computes a sample version of this population value.

\section{Skewness $S$}

The skewness are higher-order statistical attributes of a time series. Skewness indicates the symmetry of the probability density function (PDF) of the amplitude of a time series. A time series with an equal number of large and small amplitude values has a skewness of zero. A time series with many small values and few large values is positively skewed and the skewness value is positive. A time series with many large values and few small values is negatively skewed and the skewness value is negative.

$$
\mathbf{S}=\frac{E(x-\mu)^{4}}{\sigma^{4}}
$$

Where $\mu$ is the mean of signal $x, \sigma$ is the standard deviation of $\mathrm{x}$, and $E(t)$ represented the expected value of the quantity $t$ Skewness computes a sample version of this population value.

\section{Entropy E}

Entropy is a numerical measure of the randomness of a signal. Entropy can act as a feature and used to analyze psychological time series data such as EEG data. The Entropy can thus be calculated as

$$
\mathrm{E}(\mathrm{s})=\sum_{\mathrm{i}} \mathrm{E}\left(\mathrm{s}_{\mathrm{i}}\right)
$$

The $\mathrm{E}$ must be the an additive cost function such that $E(0)=0$. Entropy is the statistical descriptor of the variability within the EEG signal and is a strong feature for epilepsy detection.

\section{Median $M$}

The median is a simple measure of central tendency. To find the median, we arrange the observations in order from smallest to largest value. If there are an odd number of observations, the median is the middle value. If there is an even number of observations, the median is the average of the two middle values The median is a simple measure of central tendency. To find the median, we arrange the observations in order from smallest to largest value. If there are an odd number of observations, the median is the middle value. If there is an even number of observations, the median is the average of the two middle values.

$$
\overline{\mathrm{X}}=\frac{\sum_{\mathrm{i}=1}^{\mathrm{n}} \mathrm{X}_{\mathrm{i}}}{\mathrm{n}}
$$

$\mathrm{X}$ refers to the entire set of the numbers. Median are more robust than arithmetic mean and geometric mean if the raw data does not contain significant outliers.

\subsection{Classification}

Several statistical models have been proposed for classification and prediction. Classifying and predicting disorders based on risk factors is one of the applications of artificial neural networks $[15,16]$. Artificial neural networks are simply applicable to problems with no algorithmic solution, a complex algorithmic solution, and problems that are simple for people but difficult for computers [17]. They are also useful as an alternative solution for problems that generally have statistical solutions, such as regression 
modeling, predicting time series, cluster analysis, discriminate analysis, statistical decision-making problems, process control, and estimating the conditional distribution $[15,16]$. An artificial perceptron multi-layer neural network [18] with error back propagation algorithm was used for evaluating different states of EEG signals, such as healthy, convulsive, and epileptic states. Structure of Multilayer perceptron shown in Figure 6.

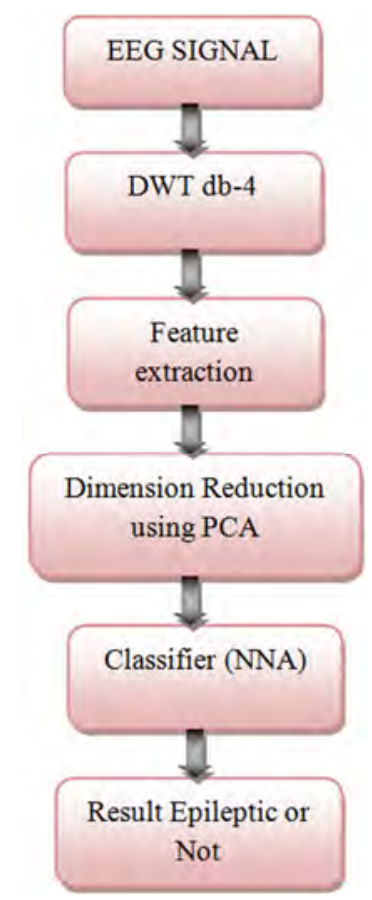

Figure 5. General flowchart for seizure classification.

In this work the dataset which is used from these data $75 \%$ data from set $\mathrm{A}$ and $75 \%$ data from set $\mathrm{E}$ is used for Training. For testing purpose $25 \%$ data is used. This selected data is used as input to the MLPNN Classifier which is used in this work. The number of neurons in the input layer symbolizes the number of features presented to the network, followed by hidden layer with neurons which transforms the input into nonlinear combinations and passes the signals to the output layer [19].

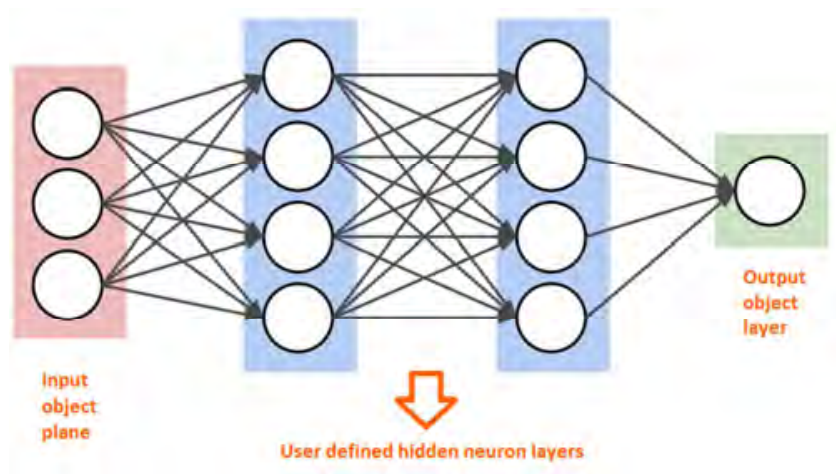

Figure 6. Multilayer perceptron.

In this proposed framework, the number of neurons in the input layer is ten corresponding to CFI and the number of neurons in the output layer is three to classify three different classes. The most challenging task is to select the number of neurons in hidden layer. In our work we varied the numbers of hidden nodes from 6 to 3 to find out the architecture giving the better performance with high accuracy. We have adapted our method by systematically enumerating all combinations of feature vectors and the combination of and checking all different size CFIs for the optimality of the features. Different features combination set has been obtained, and performance is evaluated from MLPNN.

\section{Result}

In this process classification is done for ictal state $(\mathrm{E})$ and other healthy state (A) and in order to measure the performance of the classifier, Sensitivity (True Positive Ratio - TPR) and Specificity (True Negative Ratio - TNR) were calculated.

\section{Sensitivity (TPR)}

Sensitivity is the strength of the capacity to detect and discriminate stimuli. It is how strong the perception of a stimulus is in an individual. A person who has higher sensitivity will perceive a stimulus more strongly at a lower level than someone who is less sensitive to the stimulus.

\section{Specificity (TNR)}

Specificity relates to the test's ability to correctly detect patients without a condition. Consider the example of a medical test for diagnosing a disease. Specificity of a test is the proportion of healthy patients known not to have the disease, who will test negative for it.

\section{Accuracy}

Accuracy is the degree of closeness of a measured or calculated quantity to its actual (true) value. A measurement system or computational method is called valid if it is both accurate and precise.

Equation given below describe the formula used for calculating Sensitivity and Specificity using True Positive (TP), True negative (TN), False Positive (FP) and False Negative (FN).

$$
\begin{aligned}
& \text { Sensitivity }=T P R=\frac{T N}{T P+F N} \times 100 \% \\
& \text { Specificity }=T N R=\frac{T P}{T N+F P} \times 100 \% \\
& \text { Accuracy }=\frac{T P+T N}{T P+F P+T N+F N} \times 100 \%
\end{aligned}
$$

Table 1. General Confusion matrix.

\begin{tabular}{llll}
\hline \multicolumn{4}{c}{ Predicted Class } \\
\hline & Class & Yes & No \\
\hline \multirow{2}{*}{ Actual Class } & Yes & TP & FN \\
& No & FP & TN \\
\hline
\end{tabular}

In this work obtain values of $\mathrm{TP}, \mathrm{TN}, \mathrm{FP}, \mathrm{FN}$ are $\mathrm{TP}=80, \mathrm{FP}=0, \mathrm{TN}=$ $80, \mathrm{FN}=0$. The values obtained for performance measuring Parameters is shown 


$$
\begin{gathered}
\text { Sensitivity }=\frac{80}{80+0} \times 100 \%=100 \% \\
\text { Specificity }=\frac{80}{80+0} \times 100 \%=100 \% \\
\text { Accuracy }=\frac{80+80}{80+0+80+0} \times 100 \%=100 \%
\end{gathered}
$$

Table 2. Performance.

\begin{tabular}{llll}
\hline \multicolumn{4}{c}{ Performance of ANN Classifier } \\
\hline & Accuracy & Sensitivity & Specificity \\
\hline $\begin{array}{l}\text { Normal with Eye open and } \\
\text { Epileptic classification }\end{array}$ & $100 \%$ & $100 \%$ & $100 \%$ \\
\hline
\end{tabular}

\section{Conclusion}

An expert model was developed for detection of epilepsy on the background of EEG by using discrete wavelet transform and MLPNN classifiers. It has been found that the computation time of NNA classifier is very less to provide better accuracy. The results have been shown that the proposed technique could attain a higher accuracy of $100 \%$ using classifiers for normal eyes open and epileptic seizure EEG data sets for all individual and combined statistical features derived from DWT for the detection of an epileptic seizure.

\section{Acknowledgements}

The authors are very much thankful to Dr. R. G. Andrzejak of University of Bonn, Germany, for providing permission to use the EEG data available in the public domain (R. G. Andrezejak, K. Lehnertz \& F. Morman, 2001). I owe a debt of gratitude to my project guide Dr. Arun Kumar, Associate Professor, Department of Electronics \& Telecommunication Engineering, for his right orientation, invaluable knowledge. His critical judgment and review shaped this paper to add with a quality manuscript.

\section{References}

[1] Saeid Sanei and J. A. Chambers EEG Signal Processing. John Wiley \& Sons, 2007.

[2] A. Massimo, "In Memoriam Pierre Gloor 1923-2003: an appreciation”. Epilepsia, vol. -45(7), July 2004, page-882.

[3] M. A. B. Brazier. "A History of the Electrical Activity of the Brain". The First Half-Century, Macmillan, New York, 1961.

[4] M. D. Alessandro, R. Esteller, G. Vachtsevanos, A. Hinson, A. Echauz, and B. Litt. "Epileptic seizure prediction using hybrid feature selection over multiple intracranial EEG electrode contacts: A report of four patients". IEEE Transactions on Biomedical Engineering-2003. vol. -50 (5), pp. $-603-615$.
[5] B. P. Marchant. "Time-frequency analysis for biosystem engineering". Biosystems Engineering-2003. vol. -85 (3), pp. $-261-281$.

[6] Subasi, A., EEG signal classification using wavelet function extraction and a mixture of expert model', Expert System with Application, 32, 1084-1093, 2007.

[7] A. Subasi, and M. Ismail Gursoy. "EEG signal classification using PCA, ICA, LDA and support vector machines". Expert Systems with Applications-2010. vol. 37, pp. -8659-8666.

[8] A. Subasi. "Epileptic seizure detection using dynamic wavelet network". Expert Systems with Applications-2005. vol. -29 , pp.-343-355.

[9] K. Fu, J. Qu, Y. Chai, and Y. Dong, "Classification of seizure based on the time-frequency image of EEG signals using HHT and SVM". Biomedical Signal Processing and Control-2014, vol. -13, pp. -15-22.

[10] R. G. Andrzejak, K. Lehnertz, F. Mormann, et al. "Indications of nonlinear deterministic and finitedimensional structures in time series of brain electrical activity: dependence on recording region and brain state". Phys. Rev. Ed- 64 (6)-061907.2001.

[11] Durka P. J. Adaptive time-frequency parametrization of epileptic spikes. Physical Review E 2004; 69: 051914.

[12] Kumari Pinki, and Abhishek Vaish. "Brainwave based user identification system: A pilot study in robotics environment." Robotics and Autonomous Systems 65 (2015): 15-23.

[13] C. S. Burrus, R. A. Gopinath, and H. Guo, Introduction to wavelets and wavelet transforms: A primer. Prentice-Hall, Upper Saddle River, NJ, 1998.

[14] Mandeep Singh, SunpreetKaur, Epilepsy, "Frequency Band Separation for Epilepsy Detection Using EEG", International Journal of Information Technology \& Knowledge Management, Vol 6, No. 1, Dec 2012.

[15] Livingstone D. J. Artificial Neural Networks: Methods and Applications (Methods in Molecular Biology). Humana Press, 2008.

[16] Dreiseitl S, Ohno-Machado L. Logistic regression and artificial neural network classification models: a methodology review. Journal of biomedical informatics 2002; 35: 352-359.

[17] Zini G, d'Onofrio G. Neural network in hematopoietic malignancies. Clinica chimica acta 2003; 333: 195-201.

[18] Ramchoun H, Amine M, Idrissi J, Ghanou Y, Ettaouil M. Multilayer Perceptron: Architecture Optimization and Training. International Journal of Interactive Multimedia and Artificial Inteligence. 2016; 4 (Special Issue on Artificial Intelligence Underpinning).

[19] Rezvan Abbas and Mansour Esmaeilpour International Journal of Interactive Multimedia and Artificial Intelligence, Vol. 4, No. 5, 2017: 33-38.

[20] A. Sharmila P. Geethanjali "DWT-Based Detection of Epileptic Seizure from EEG Signals" IEEE Transuction on Digital Object Identifier vol. 1 2016, pp- 7716 - 7727. 